The results indicate the importance of the presence of the two reacting hydrogen atoms in the 9-position, in combination with the amino group in the 2-position, in determining the growth-inhibiting and possibly the carcinogenic activity of 2 -aminofluorene.

The possibility of studying the action of a chemical carcinogen by its effect on bacterial metabolism has obvious advantages in the analysis of the mechanism involved.

F. BielschowsKy.

H. N. Green.

Department of Pathology, and Cancer Research Laboratories,

University of Sheffield. April 16.

'Wilson, R. H., DeEds, Floyd, Cox, A. J., Cancer Research, 1, 595 (1941).

\section{Time of Collapse of a Soap Bubble}

A SPHERICAI soap bubble of radius $R$ blown at the end of a uniform glass tube of radius $a$ and length $l$ will gradually deflate itself and disappear. Its time of collapse $\tau$ can be computed on the assumption that the gas inside the bubble of viscosity $\eta$ passes out of the tube in streamline flow under the pressure excess in the bubble. In a spherical soap bubble of radius $r$, this pressure excess is $4 T / r$, where $T$ is the surface tension of the soap solution. Hence from a consideration of the viscous flow of a gas in a tube under a small pressure difference between its ends, it follows that,

whence

$$
-d\left(\frac{4}{3} \pi r^{3}\right)=\frac{\pi a^{4}}{8 \eta 7} \frac{4 T}{r} d t ;
$$

$$
\text { Hence } \frac{R^{4}}{\tau}=\frac{-\int_{a^{4}}^{0}}{2 \eta l}{ }_{R}^{3} d r=\int_{0}^{\tau} \frac{T a^{4}}{8 \eta l} d t .
$$

It has been found experimentally possible to blow spherical soap bubbles of more than $5 \mathrm{~cm}$. diameter, the shape of which, in spite of gravity, can be considered to be spherical to a good approximation. When the bubbles are suitably blown, the vertical and horizontal diameters do not differ by more than a quarter per cent even in large bubbles of about $8 \mathrm{~cm}$. diameter. The experimental results have confirmed the above relation, which therefore opens up a new method of estimating the surface tension of different soap solutions. Under suitable experimental conditions, a determination of the viscosities of certain gases or their effect on the surface tension of soap films is also rendered possible.

Experimental results will soon be published else. where.

L. Sibatya.

University of Mysore,

Bangalore.

Jan. 12.

\section{Enzymatic Synthesis of Levan}

STUDY of the formation of micro-organisms of polysaccharides from sucrose has been hampered by the absence of a satisfactory technique for the cellfree isolation of the enzyme catalysts concerned ${ }^{1}$. This obstacle has now been removed. The separation of a dextran-synthesizing enzyme from Leuconostoc mesenteroides has been reported recently by $\mathrm{Hehre}^{2}$ It is the purpose of the present note to report the isolation from Bacillus subtilis and Aerobacter of an enzyme or enzymes which act on sucrose with formation of levan.

In sucrose-agar, colonies of Bacillus subtilis induce formation of levan to some distance from their site of growth ${ }^{3}$. When cell-free agar pieces from the vicinity of such $B$. subtilis colonies are aseptically removed to sterile sucrose solutions, they cause the gradual appearance in the fluid of a pronounced opalescence accompanying the formation of a polysaccharide which is identical in chemical and physical properties with levan. After 24 hours incubation, the amount of polysaccharide formed was considerable (300 mgm./per cent). When ground up in sucrose solution, a partial extraction of synthesizing enzyme from its agar matrix was obtained. This technique proved ill-suited, however, to the isolation of the synthesizing enzyme on a considerable scale.

A further and more convenient source of a levansynthesizing enzyme was found in a levan-forming strain of Aerobacter. This organism, unlike most of the aerobic spore formers, is easily killed by narcotics. Cell-free autolysates prepared from an aqueous suspension of the cells by incubation with a little thymol in chloroform and subsequent centrifugation actively synthesized levan from sucrose (393 mgm. per cent in $40 \mathrm{hr}$.). On drying over Drierite in vacuum a stable water-soluble powder of considerable synthetic activity was obtained.

Control tests showed definitely that synthesis by the different enzyme preparations which have been described had occurred in the complete absence of living bacterial cells.

It seems reasonable to hope that with the aid of these enzyme preparations, new insight will be obtained into the sequence of reactions leading to levan formation. A full report on the properties and activity of the levan-synthesizing enzyme preparations will be presented elsewhere.

\section{ASChNer. \\ S. Avinert-Shapiro. \\ S. Hestrin.}

Dept. of Hygiene and Bacteriology, and Chemistry Dept., Cancer Laboratories, Hebrew University, Jerusalem.

Feb. 15.

${ }^{1}$ Harrison, Tarr and Hibbert, Can. J. Res., 3, 499 (1930).

${ }^{2}$ Hehre, Science, 93, 237 (1941).

${ }^{3}$ Beijerinck, Folia Microbiologica, 1, 377 (1912).

\section{Meiosis in Drosophila}

UP to now students of Drosophila have regarded meiosis in the male as almost inaccessible, and have, therefore, confined themselves to the genetical investigation of essentially cytological problems. This is due to the belief that meiosis was completed in the pupa and that only the actual spermiogenesis takes place in the adult.

I have worked principally with aceto-orcein smear preparations of testes of $D$. subobscura. Meiosis in this species starts in the fully grown larva and continues in waves throughout the whole of pupal and adult life at least up to a fortnight after eclosion. It subsides during periods of differentiation, for example, when the vasa deferentia begin to grow, and during periods of great stress, for example, on emergence, so that no divisions occur in young males up to two days of age.

In view of these facts Huettner's statement ${ }^{1}$ that 